\title{
Zermelo-Markov-Dubins problem and extensions in marine navigation*
}

\author{
Jean-Baptiste Caillau ${ }^{1}$ Sofya Maslovskaya ${ }^{2}$ Thomas Mensch ${ }^{3}$ Timothée Moulinier ${ }^{3}$ Jean-Baptiste Pomet ${ }^{2}$
}

\begin{abstract}
This note accounts for optimal control techniques applied to marine navigation for seismic acquisition. More precisely, the goal is to gain time in turns and alignment maneuvers. A model for the kinematics of the marine vessel and sea current is proposed, then extended to include the evolution of the shape of the towed underwater cables during the maneuver. Two minimum time problems are stated, depending on whether the shape of the streamers is in the model or not. The simpler case is the so-called Zermelo-Markov-Dubins problem, recently studied in the literature. It generalizes the classical Dubins problem. The complete model is not standard, and preliminary analysis of controllability and of properties of minimum time trajectories are given.
\end{abstract}

\section{INTRODUCTION}

The motivation for the present work is to optimize turns and maneuvers of marine vessels towing a set of long and fragile underwater cables. This motivation coming from marine seismic acquisition is described in details in Section [II From an optimal control point of view, this has previously been set as a Dubin's problem [6]: the magnitude of the speed being fixed, one seeks the shortest path from a point to another (including direction of the tangent) with a bound on curvature. The maximum curvature has to be small enough in order to preserve the integrity of the towed equipment during the turn. There are two drawbacks to this approach: it does not take into account possible sea currents, and it does not contain any description of the hydrodynamic behavior of the towed cables (the dynamic equations only contain a kinematic of the ship itself). Adding the sea current into the problem, without modelling the cable behavior, leads to a so-called Zermelo-Markov-Dubins problem (the term was coined in [2]), i.e. a Dubin's problem with a drift. Both optimal control problems lead to singular curves that are straight lines and bang-bang curves that are circles in the case of Dubin's problem and trochoids in the case of ZermeloMarkov-Dubins problem. See [6] for the original solution of Dubin's problem. It has then been revisited in terms of optimal control using Pontryagin Maximum Principle, see [3], [11]. Zermelo-Markov-Dubins problem appeared in the first place from airplane path planning with constant wind [9], it is also well documented in [2] and [12].

\footnotetext{
*This work was partly supported by CGG under a research contract.

${ }^{1}$ Jean-Baptiste Caillau is with Université Côte d'Azur, CNRS, Inria, LJAD, France jean-baptiste.caillau@univ-cotedazur.fr

${ }^{2}$ Sofya Maslovskaya and Jean-Baptiste Pomet are with Université Côte d'Azur, Inria, CNRS, France sofya.maslovskayalinria.fr, jean-baptiste.pomet@inria.fr

${ }^{3}$ Thomas Mensch and Timothée Moulinier are with Marine Acquisition Division of CGG, Massy, France thomas.mensch@cgg.com, timothee.moulinier@cgg.com.
}

In this paper, we propose a model for the vessel including dynamics of the towed cables and a preliminary analysis of the associated minimum time problem. We do not know of any previous attempt in this direction. Note that the Zermelo-Markov-Dubins problem can be seen as minimum time for a "unicycle" with constant speed magnitude and bounded turning rate, where rolling without sleeping holds with respect to a horizontal plane moving with constant speed. Our model presented here adds a "trailer" to this unicyle. See [8], [10] for unicycles, cars and trailers. To the best of our knowledge, even without the constant slip, minimum time for Dubin's problem with one or more trailers has no yet been solved.

The paper is organised as follows. Section [II details the marine acquisition problem and its setting in optimal control terms. Section [II] describes the kinematic models behind the Zermelo-Markov-Dubins problem as well as the one where the towed streamers are explicitly taken into account. Section IV gives some insights on the obtained control system and preliminary results on controllability. Section $\mathrm{V}$ finally gives an analysis of the obtained extremal flow for the system with one trailer.

\section{THE MARINE NAVIGATION PROBLEM}

Marine seismic acquisition aims at collecting data to image the subsurface of the Earth. It is done by deploying seismic sources and a large number of pressure sensors, distributed along underwater cables (streamers); up to 14 streamers each separated by $100 \mathrm{~m}$ and possibly $10 \mathrm{~km}$ long are towed between 7 and $50 \mathrm{~m}$ below the sea surface. An example of seismic spread is displayed Figure 1. At short time intervals, the seismic sources emit an acoustic signal which propagates in the subsurface so that hydrophones located along the cable record the resulting reflected wave field. These data are then used to build structural images of the subsurface and to delineate possible oil and gas reservoirs. During marine surveys, the seismic vessel sails parallel acquisition lines back and forth, at low speed, in order to cover the survey area. In production, the typical vessel speed in water is around 5 knots, that is about $2.5 \mathrm{~m} / \mathrm{s}$. (Limitations are due to limits to the maximum working load of the towed spread and to the power of the vessel engine.) At the end of an acquisition line, the vessel typically performs a large $180^{\circ}$ turn maneuver (U-turn) followed by a straight "run-in" segment in order to start the next acquisition line with the towed cables aligned. Figure 2 illustrates a typical acquisition pattern for a seismic survey (see [7] for more details about seismic acquisition). Obviously there are strict geographical constraints (following predefined lines) on the 
vessel during production lines. On the contrary, during the turn, that we may also call the line change, the trajectory is free, constrained only at start and end times where the ship has to be at specific geographical points with a given sailing direction (and ideally a given configuration of the streamers). This is why there is room for optimizing the duration of this phase: reducing this duration may significantly benefit to the survey productivity by reducing non-productive time.

The optimal control problem will then be minimum time. Let us describe dynamics and constraints. The usual setting is to set a maximum turn rate (or minimum turn radius) of the vessel during the line change, chosen small enough to preserve the spread integrity during the turn with regards to mechanical and hydrodynamic equipment limitations; it depends mainly on the size of the seismic spread (i.e. width of the spread, length and number of the towed cables); typically the minimum turn radius for a seismic vessel towing equipment in water is between 4 and $5 \mathrm{~km}$. The minimum

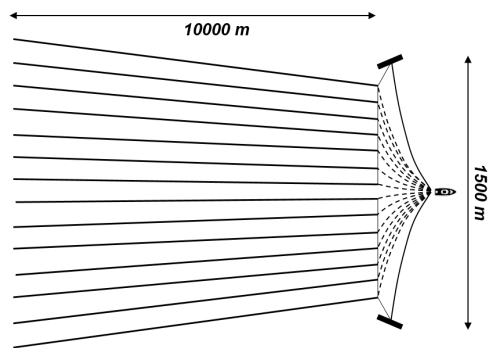

Fig. 1. Example of seismic acquisition spread towed by a vessel. The equipment at sea consists in seismic sources towed behind the seismic vessel and long streamers containing the hydrophones spread with large vanes.

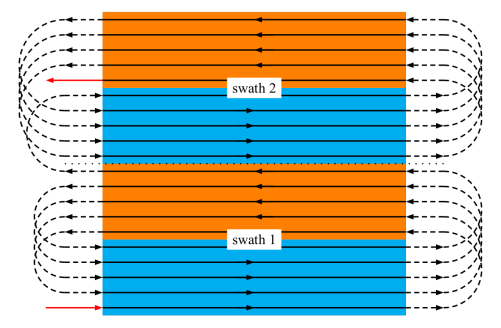

Fig. 2. Typical acquisition pattern for a seismic survey. The sequence of acquired lines is chosen for minimizing the line change duration assuming no sea current and a given minimum turn radius.

time problem described so far is Dubins problem [6]. Optimal path candidates are made of straight lines and circle arcs, and computing the correct succession between given initial and final conditions is straightforward. It is the method used for pre-planning the line changes prior to the survey (see Figure 2). In absence of sea current, this seems sufficient. Most of the time, whatever the ocean current conditions are, the turning path is described with respect to the ground as a sequence of straight lines and arc circles (Dubins path). However, in the case of strong sea current conditions this type of line changes may not be adapted both for survey efficiency and in-water equipment safety reasons. Equipment mechanical limitations are related to the speed and to the turning rate with respect to the fluid rather than the ground, and when the current is strong, they differ a lot. Writing that the turning rate bound is satisfied with respect to a frame moving with the fluid leads to Dubin's paths in that frame, or to the optimal control problem that we called ZermeloMarkov-Dubins in the introduction and further. One last consideration is that, as mentioned above (Figure 2, a "run in" phase (typically one streamer long) is usually added to re-align the streamer because the optimization does not take its shape into account. A way to reduce, and ideally remove, the run-in phase is to include its shape in the optimal control problem, adding a constraint on the shape of the towed cable (and its stability) to the optimization. The model presented in section $\amalg I-B$ and the elements towards a solution of the associated minimum time problem presented in Section $\mathrm{V}$ go in this direction.

\section{MODEL}

We consider a ship moving in a portion of ocean undergoing a constant current (the whole fluid has constant horizontal velocity $W$ ) and towing a spread. Let us first model the movement of the ship alone, then of the ship with its towed streamer.

\section{A. A ship in water with current}

Fix a reference frame, attached to the Earth, and call "water frame" the frame that moves with constant velocity $W$ and coincides with the fixed frame at time zero. Denoting by $(x, y)$ the coordinates of the ship in the reference frame, its coordinates in the water frame are $\left(x-t W_{x}, y-t W_{y}\right)$, where $W_{x}, W_{y}$ are the coordinates of $W$. Define $v$ and $\theta$, the magnitude and polar angle of the ship's velocity with respect to the water frame, as follows:

$$
\left(\begin{array}{l}
\dot{x}-W_{x} \\
\dot{y}-W_{y}
\end{array}\right)=v\left(\begin{array}{c}
\cos \theta \\
\sin \theta
\end{array}\right) .
$$

We use a very simple kinematic model in which $v$ and $\dot{\theta}$ are considered as inputs, i.e. they can be assigned. The real controls are the rudder angle and the thrust of the engine, hence we have not taken into account the dynamic effects, like the dynamics of the ship itself; this is legitimate because, given a prescribed path $t \mapsto(x(t), y(t))$, some lower level control loops (auto-pilot) are in charge of controlling the engine and rudders to produce that precise prescribed path. Here we idealize these control loops. We constrain the two controls $v$ and $\dot{\theta}$ as follows:

$$
v>0 \text { is constant, } \quad|\dot{\theta}| \leq \rho
$$

where $\rho$ is a positive number to be determined later. Defining $u$ as $\dot{\theta}=u$, one gets a control system with state $(x, y, \theta)$ and control $u$ that depends on the two positive numbers $\rho, v$ and the vector $W$ as parameters. Let us normalize these parameters. Re-scaling time and length, one easily normalizes $v$ and $\rho$ to 1 . Then, rotating the reference frame so that its $(O x)$ axis is aligned in the direction of $W$ puts its second component $W_{y}$ to zero and renders its first component 
$W_{x}$ positive. Gathering all the above, we get the following normalised control system:

$$
\dot{x}=w+\cos \theta, \dot{y}=\sin \theta, \dot{\theta}=u, \quad|u| \leq 1,
$$

where $w$ is a non-negative number. We impose

$$
0 \leq w<1
$$

because $w \geq 1$ would mean that the current is more powerful than the ship, and clearly would make the system noncontrollable because $x$ could never decrease. With $z=$ $x+i y$, one may write (3) in complex notations:

$$
\dot{z}=w+e^{i \theta}, \quad \dot{\theta}=u, \quad|u| \leq 1 .
$$

\section{B. Modeling long streamers towed by the ship}

The ship now tows a streamer. A precise model of this deformable attachment would be a distributed one, governed by PDEs, including some fluid dynamics and some inner forces and couples. Such models account rather precisely for the real behavior, they are suitable for simulations, but we seek a less complex one, to be used for control design. The simplest possibility is to depict the system as a chain of elements that interact punctually with the fluid - simplest interaction: a drag proportional to the speed with respect to the fluid - and are joined by links that keeps distances constant. As a first step, we consider the case of only one such element. For lack of a better term, call this point a bead. Figure 3 depicts the geometry of this simplified model that is merely the ship plus one bead.

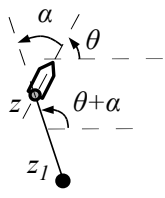

Fig. 3. The ship and its simplified streamer

Ordinary differential equations can be rather readily derived. One first assumption, that is also made when using PDE models for the streamers, is that the dynamics of the ship itself is not affected by the streamers, see more justification right after (7). Hence equations (3) or (5) are still valid. If the position of the bead is represented by a complex number $z_{1}$, the distance constraint imposed by the link is $\left|z-z_{1}\right|=\ell$. We define the angle $\alpha$ by

$$
z_{1}=z-\ell e^{i(\theta+\alpha)} \text {. }
$$

There are two forces applied to $z_{1}$ : the tension from the link, co-linear to the link, hence equal to $T e^{i(\theta+\alpha)}$ with $T$ a positive real number, and the drag, given by $-k\left(\dot{z}_{1}-w\right)$ for some constant $k>0$. Dynamic equations hence read

$$
m \ddot{z}_{1}=T e^{i(\theta+\alpha)}-k\left(\dot{z}_{1}-w\right) .
$$

We make here the assumption of a quasi-static movement, i.e. the mass of $z_{1}$ is small enough that the limit speed is always attained immediately so that the equation becomes

$$
T e^{i(\theta+\alpha)}-k\left(\dot{z}_{1}-w\right)=0 .
$$

This assumption amount to say that the inertia of the towed engine is very small compared to the drag forces. It is consistent with the assumption that the speed of the ship can be seen as a control, as explained in Section III-A. Getting $\dot{z}_{1}$ from (6) and (5), substituting in (7) and equating real and imaginary parts yields

$$
\dot{\alpha}=-\frac{1}{\ell} \sin \alpha-u, \quad T=\rho \cos \alpha .
$$

The second equation gives the tension, it is not needed to write the dynamics, but it reveals that $\alpha$ must always satisfy

$$
-\frac{\pi}{2}<\alpha<\frac{\pi}{2}
$$

because $T$ has to be positive; indeed, the link between the ship and the bead (in fact the whole spread of streamers) has to be in positive tension, or else the ship would "push" the streamer, as if the link was a rod.

We arrive to the following control system:

$$
\dot{x}=w+\cos \theta, \quad \dot{y}=\sin \theta, \quad \dot{\theta}=u, \quad \dot{\alpha}=-\frac{\sin \alpha}{\ell}-u,
$$

or, with complex notations

$$
\dot{z}=w+e^{i \theta}, \quad \dot{\theta}=u, \quad \dot{\alpha}=-\frac{\sin \alpha}{\ell}-u,
$$

where the control $u$ is constrained by

$$
|u| \leq 1,
$$

and the two parameters $w$ and $\ell$ satisfy

$$
0 \leq w<1, \quad 0<\ell<1 .
$$

The state space is $\mathbb{C} \times S^{1} \times(-\pi / 2, \pi / 2)$ (we use the notation $\mathbb{C}$ even when using $(x, y)$ as coordinated). It is invariant in positive time (for any control).

Remark 1 (alternative view of the same model). In the water frame, the equations of the ship are the ones of a "rolling penny", or a "unicycle": $\theta$ is the direction of the axis of the vehicle and its speed is parallel to this axis. Now assume that $z_{1}$ is the center of the axle of a trailer attached to the unicycle at point $z$. Writing that the distance between $z$ and $z_{1}$ is constant equal to $\ell$ yields (6). Writing that the trailer is rolling without slipping in the water frame amounts to saying that the speed of $z_{1}$ with respect to the water frame, equal to $e^{i \theta}-\ell i(u+\dot{\alpha}) e^{i(\theta+\alpha)}$ according to (6), is aligned with the line from $z_{1}$ to $z$, i.e. with $e^{i(\theta+\alpha)}$. This yields the same equation for $\dot{\alpha}$ as in $(10)$. We just showed that the model we wrote is also the one of a unicycle with one trailer (as described in [8] or references therein) where the rolling without slipping is achieved in a frame that moves at constant speed 1 in the reference frame, $w$ may be viewed as a constant slip. Note that this analogy no longer holds when one considers more than one element, i.e. when the spread of streamers is modeled by two or more of these "floating beads".

\footnotetext{
${ }^{1}$ Best picture: a unicycle with one trailer rolling without slipping on a conveyor belt.
} 
Because of the above remark, we often use, in the next sections, the term "trailer" for the attachment represented by the point $z_{1}$ in Figure 3 that figures the spread of streamers towed by the ship.

\section{Minimum time problem}

The simplest way to set an optimal control problem is on system (5) (or (3)) alone. We are then seeking, for fixed $\left(z_{0}, \theta_{0}\right)$ and $\left(z_{f}, \theta_{f}\right)$, the smallest positive $T$ such that there is a solution of (5) such that $z(0)=z_{0}, z(T)=z_{f}$, $\theta(0)=\theta_{0}$ and $\theta(T)=\theta_{f}$. The bound $|u| \leq 1$ is present to ensure integrity of the spread of streamers: it is a bound on the curvature of the trajectory observed in the water frame, i.e. the one that really affects the vessel in its interactions with the fluid. The optimal control problem is known as the Zermelo-Markov-Dubins problem [9], [12], [2], already mentioned in the introduction.

We noted in section II that, in general, at the end of the turn, the streamer does not have the stable configuration required for acquisition, and this is corrected by adding to the turn another phase (run-in) where the vessel sails along a straight line to re-align the streamer to the stable shape that it has when sailing straight in the following acquisition line. In order to include realignment in the turn, we have given a simple model of the streamer, represented by the angle $\alpha$. In the model, straight lines correspond to $u=0$, and clearly, from the equation for $\alpha$, the stable position is $\alpha=0$, i.e., if one wants to gain the time this angle takes to go to zero at the end of the turn, it is more efficient to set up a minimum time problem for system (11) rather than (5), with a desires final condition specifying $\alpha=0$. We give in Section $\mathrm{V}$ preliminary results on the minimum time problem for 111 with a final condition $\left(z_{f}, \theta_{f}, 0\right)$. We note in the next section that, anyway, large values of $\alpha$ are in general not reachable.

\section{Controllability}

Let us define the set of points reachable from a given point.

Definition 2 (reachable sets for (11)-(12)). For any $\left(z_{0}, \theta_{0}, \alpha_{0}\right)$ in $\mathbb{C} \times S^{1} \times\left(-\frac{\pi}{2}, \frac{\pi}{2}\right)$, let $\mathrm{A}^{+}\left(z_{0}, \theta_{0}, \alpha_{0}\right)$ be the set of points $\left(z_{1}, \theta_{1}, \alpha_{1}\right)$ in $\mathbb{C} \times S^{1} \times\left(-\frac{\pi}{2}, \frac{\pi}{2}\right)$ such that there exists a positive number $T$ and a solution $(z(\cdot), \theta(\cdot), \alpha(\cdot), u(\cdot))$ of (11)-(12) satisfying $(z(0), \theta(0), \alpha(0))=\left(z_{0}, \theta_{0}, \alpha_{0}\right)$ and $(z(T), \theta(T), \alpha(T))=\left(z_{1}, \theta_{1}, \alpha_{1}\right)$.

Denote by $\alpha^{*}$ the angle in $(0, \pi / 2)$ such that $\ell=\sin \alpha^{*}$. Notice that as $\ell<1$, such an angle exists. The angle $\alpha^{*}$ is an asymptotically stable equilibrium of $\dot{\alpha}=1-(\sin \alpha) / \ell$ which corresponds to the dynamics of $\alpha$ in (11) associated with control $u \equiv-1$. Symmetrically, $-\alpha^{*}$ is an asymptotically stable equilibrium of $\dot{\alpha}$ with $u \equiv 1$. In the case with trailer, system (11)-12 has the following important property.

Proposition 3 (Non controllability). For any $(z, \theta, \alpha)$ in $\mathbb{C} \times$ $S^{1} \times\left(-\alpha^{*}, \alpha^{*}\right)$, one has

$$
\mathrm{A}^{+}(z, \theta, \alpha) \subset \mathbb{C} \times S^{1} \times\left(-\alpha^{*}, \alpha^{*}\right) .
$$

This defeats global controllability for (11)-(12): a point that is not in the domain $\mathbb{C} \times S^{1} \times\left(-\alpha^{*}, \alpha^{*}\right)$ cannot be reached from it.

Let us return to our problem in marine navigation. The constraints of the marine seismic acquisition problem which motivated our studies suggest that not all states in $\mathbb{C} \times S^{1} \times$ $\left(-\alpha^{*}, \alpha^{*}\right)$ need to be reached and only the states with the stable configuration of the trailer (spread of streamers) are important, i.e. the states at which the vessel may start an acquisition line. In our model it can be translated in terms of the angle $\alpha$. The stable configuration means that the control $u$ is set to be zero and the final value of $\alpha$ should be an equilibrium of $\dot{\alpha}=-\frac{1}{\ell} \sin \alpha$, which implies $\alpha=0$. Therefore, the important problem to study is the reachability of final states with $\alpha=0$. For this kind of final states we have the following result.

Theorem 4. For any $\left(z_{0}, \theta_{0}, \alpha_{0}\right) \in \mathbb{C} \times S^{1} \times(-\pi / 2, \pi / 2)$ and any $\left(z_{f}, \theta_{f}\right) \in \mathbb{C} \times S^{1}$ there exists a control $u$ such that the corresponding trajectory of (11)-(12) joins $\left(z_{0}, \theta_{0}, \alpha_{0}\right)$ to $\left(z_{f}, \theta_{f}, 0\right)$.

See our forthcoming paper [13] for the proof of the statements above as well as results on the controllability in the case without trailer (controllability for Zermelo-MarkovDubins problem).

\section{EXTREMAL FLOW}

We give a preliminary analysis of the extremal trajectories of the minimum time problem. Namely, we prove that the extremal flow is integrable, which is an important step in the search for the optimal synthesis. We consider the fourdimensional dynamics in form 10 with state $(x, y) \in \mathbb{R}^{2}$, $\theta \in S^{1}$ and $\alpha \in(-\pi / 2, \pi / 2)$. The Hamiltonian of the system writes

$$
H=p^{0}+p_{x}(w+\cos \theta)+p_{y} \sin \theta+\left(p_{\theta}-p_{\alpha}\right) u-\frac{p_{\alpha}}{\ell} \sin \alpha,
$$

where $p^{0}$ and $p=\left(p_{x}, p_{y}, p_{\theta}, p_{\alpha}\right)$ denote the adjoint variables to the cost (final time) and state, respectively. We assume that endpoints conditions are prescribed and recall that the scalar control is bounded, $|u| \leq 1$, while the two parameters of the problem verify $0 \leq w<1$ and $0<\ell<1$.

Theorem 5 (Pontrjagin maximum principle, [1]). If $u$ is a time minimizing control, there exists a non-trivial couple $\left(p^{0}, p\right), p_{0} \leq 0$ and $p$ absolutely continuous such that, if $q$ is the associated trajectory and $t_{f}$ the minimum final time, almost everywhere on $\left[0, t_{f}\right]$

$$
\dot{q}=\frac{\partial H}{\partial p}, \quad \dot{p}=-\frac{\partial H}{\partial q}
$$

while

$$
0=H(q(t), p(t), u(t))=\max _{v \in[-1,1]} H(q(t), p(t), v)
$$


The adjoint system is

$$
\begin{aligned}
\dot{p}_{x} & =0, \\
\dot{p}_{y} & =0, \\
\dot{p}_{\theta} & =p_{x} \sin \theta-p_{y} \cos \theta, \\
\dot{p}_{\alpha} & =\frac{p_{\alpha}}{\ell} \cos \alpha,
\end{aligned}
$$

so $\left(p_{x}, p_{y}\right)$ is a constant vector. We note that either $p_{\alpha}$ is identically zero, or it never vanishes. Because of transversality conditions, the case $p_{\alpha} \equiv 0$ occurs in particular when the final value of the angle $\alpha$ is left free; then, it is clear from the expression of the Hamiltonian that the system is reduced to the dimension three Zermelo-Markov-Dubins problem studied in [12], the angle $\alpha$ evolving independently of the rest of the state coordinates. For this problem, it is shown that, for distant enough initial and final points in $\mathbb{R}^{2}$, the optimal solution is bang-singular-bang, singular arcs being straight lines as in Dubins problem. (See [12] for a complete account.) We focus on the case $p_{\alpha} \neq 0$ in the rest of the section, assuming $p_{\alpha}>0$ (the case $p_{\alpha}<0$ is symmetric). We classify the extremals according to whether the constant vector $\left(p_{x}, p_{y}\right)$ is zero or not.

a) Case $\left(p_{x}, p_{y}\right)=(0,0)$. We show that in the considered domain only bang arcs are possible, that is arcs along which the switching function $p_{\theta}-p_{\alpha}$ does not vanish identically. Along such arcs, the maximization condition implies that the control is equal to \pm 1 , depending on the sign of the switching function.

Proposition 6. If $\left(p_{x}, p_{y}\right)=(0,0)$, there are no singular arcs in the domain $\alpha \in(-\pi / 2, \pi / 2)$.

Proof. Assume by contradiction that $p_{\theta}=p_{\alpha}$ on a nonempty time interval. Since $\left(p_{x}, p_{y}\right)=(0,0)$, the adjoint state $p_{\theta}$ is constant, and so is $p_{\alpha}$. Then $0=\dot{p}_{\alpha}=\left(p_{\alpha} / \ell\right) \cos \alpha$, which is impossible for $p_{\alpha}>0$ and $\alpha \in(-\pi / 2, \pi / 2)$.

b) Case $\left(p_{x}, p_{y}\right) \neq(0,0)$. We normalize the pair $\left(p^{0}, p\right)$ according to $p_{x}^{2}+p_{y}^{2}=1$ and set $p_{x}=\cos \varphi, p_{y}=\sin \varphi$. In this case, on the basis of the analysis for the 3-dimensional model without trailer, one can expect that singular trajectories exist and play an important role in the synthesis. Set $q:=(x, y, \theta, \alpha)$, so that the controlled dynamics 10$)$ writes $\dot{q}=F_{0}(q)+u F_{1}(q)$ with

$$
F_{0}=(w+\cos \theta) \frac{\partial}{\partial x}+\sin \theta \frac{\partial}{\partial y}-\frac{1}{\ell} \frac{\partial}{\partial \alpha}
$$

and

$$
F_{1}=\frac{\partial}{\partial \theta}-\frac{\partial}{\partial \alpha} .
$$

Denoting $H_{i}=\left\langle p, F_{i}(q)\right\rangle$ the Hamiltonian lifts of these vector fields, the switching function is $H_{1}=p_{\theta}-p_{\alpha}$ and its derivative wrt. to time along a singular arc vanishes. As $\dot{H}_{1}=\left\{H, H_{1}\right\}=\left\{H_{0}, H_{1}\right\}$ using Poisson brackets, one has $H_{1}=H_{01}=0$ along such an arc (with obvious notation $H_{01}:=\left\{H_{0}, H_{1}\right\}$, and so forth). Differentiating again, one gets $H_{001}+u H_{101}=0$. Computing,

$$
H_{01}=\sin (\theta-\varphi)-\frac{p_{\alpha}}{\ell} \cos \alpha, \quad H_{001}=-\frac{p_{\alpha}}{\ell^{2}},
$$

and

$$
H_{101}=\cos (\theta-\varphi)-\frac{p_{\alpha}}{\ell} \sin \alpha .
$$

Using $H=0$ and setting $\gamma:=-p^{0}-p_{x} w$, one actually obtains $H_{101}=\gamma$. The generalized Legendre-Clebsch condition states that $H_{101}$ is nonnegative, and there are two cases: either the constant $\gamma$ is positive, or $\gamma=0$ (implying that singular arcs, if any, would be of order at least two). Now,

$$
0=\dot{H}_{01}=-\frac{p_{\alpha}}{\ell^{2}}+\gamma u,
$$

so neither $\gamma$ nor $u$ can vanish as $p_{\alpha}$ is positive. We have proven the following.

Proposition 7. If $\left(p_{x}, p_{y}\right) \neq(0,0)$, the constant $\gamma$ is positive and singular arcs are of order one. The singular control is $u_{s}=p_{\alpha} /\left(\gamma \ell^{2}\right)$.

Plugging this singular control into the original Hamiltonian defines the singular Hamiltonian

$H_{s}=p^{0}+p_{x}(w+\cos \theta)+p_{y} \sin \theta+\left(p_{\theta}-p_{\alpha}\right) \frac{p_{\alpha}}{\gamma \ell^{2}}-\frac{p_{\alpha}}{\ell} \sin \alpha$.

Defining

$$
\Sigma:=\left\{H_{1}=0\right\}, \quad \Sigma^{\prime}:=\left\{H_{1}=H_{01}=0\right\},
$$

the following is standard (see, e.g., [4]).

Lemma 8. The submanifold $\Sigma^{\prime}$ is invariant by the flow of $H_{s}$, and on $\Sigma^{\prime}$ extremals are integral curves of $H_{s}$.

On singular arcs, one has

$$
\dot{\alpha}=-\frac{p_{\alpha}}{\gamma \ell^{2}}-\frac{\sin \alpha}{\ell}, \quad \dot{p}_{\alpha}=\frac{p_{\alpha}}{\ell} \cos \alpha .
$$

Rescaling time according to $t=\ell s$, and setting $\lambda:=$ $p_{\alpha} /(\gamma \ell)$, this subsystem can be written independently of $\ell$ (with' $=\mathrm{d} / \mathrm{d} s$ )

$$
\alpha^{\prime}=-\lambda-\sin \alpha, \quad \lambda^{\prime}=\lambda \cos \alpha .
$$

Lemma 9. Subsystem 15) is Hamiltonian on $T^{*} S^{1}$ and has four equilibria: two saddles at $(0,0)$ and $(\pi, 0)$ and two centers at $(\pi / 2,-1),(-\pi / 2,1)$. (See Figure 4 for the corresponding phase portrait.)

Proof. A suitable Hamiltonian is $-\lambda(\lambda / 2+\sin \alpha)$.

Proposition 10. The singular flow is Liouville integrable.

Proof. The previous lemma allows to integrate coordinates $\left(\alpha, p_{\alpha}\right)$, while the subsystem $\left(\theta, p_{\theta}\right)$ is also Hamiltonian on $T^{*} S^{1}$ for

$$
\cos (\theta-\varphi)+\frac{p_{\theta}^{2}}{2 \gamma \ell^{2}} .
$$

An additional integration suffices to retrieve $(x, y)$.

On the open complement of the codimension one switching surface $\Sigma$, the control is bang and one can define the 


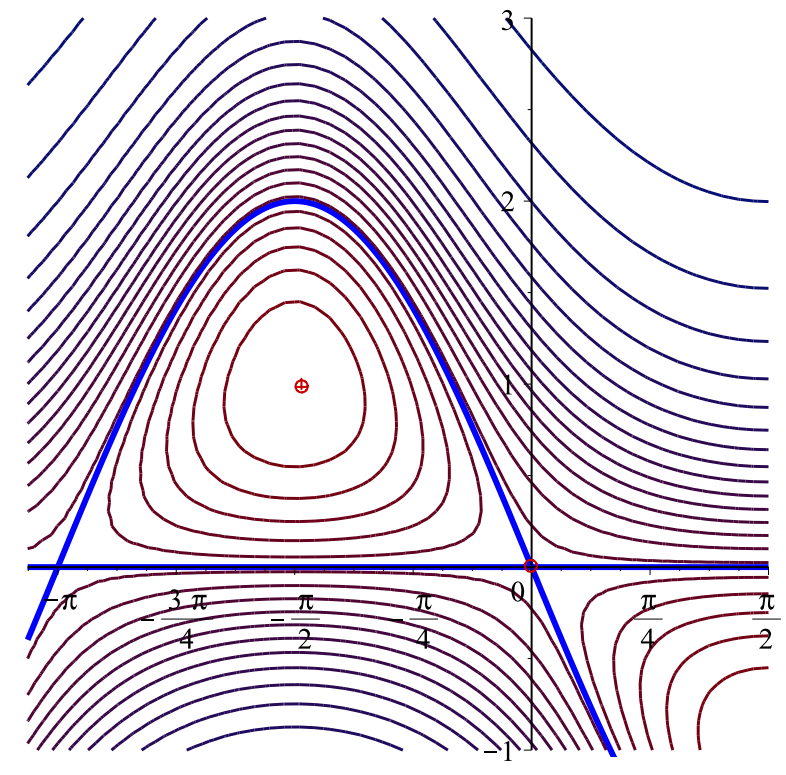

Fig. 4. Phase portrait of the Hamiltonian subsystem 15. Coordinates $(\alpha, \lambda) \in T^{*} S^{1}$.

maximized (or regular) Hamiltonian by plugging into $H$ the value $u=\operatorname{sign}\left(p_{\theta}-p_{\alpha}\right)$ :

$H_{r}=p^{0}+p_{x}(w+\cos \theta)+p_{y} \sin \theta+\left|p_{\theta}-p_{\alpha}\right|-\frac{p_{\alpha}}{\ell} \sin \alpha$.

The following readily holds.

Lemma 11. Outside $\Sigma$, extremals are integral curves of the regular Hamiltonian $H_{r}$.

On regular arcs, one has $u= \pm 1$ (constant) and

$$
\dot{\alpha}=-u-\frac{\sin \alpha}{\ell}, \quad \dot{p}_{\alpha}=\frac{p_{\alpha}}{\ell} \cos \alpha .
$$

Rescaling as before according to $t=\ell s$ and $\lambda:=p_{\alpha} /(\gamma \ell)$, the subsystem can be written (with ${ }^{\prime}=\mathrm{d} / \mathrm{d} s$ )

$$
\alpha^{\prime}=-\ell u-\sin \alpha, \quad \lambda^{\prime}=\lambda \cos \alpha .
$$

Lemma 12. Subsystem (16) is Hamiltonian on $T^{*} S^{1}$ and has two saddle equilibria: $\alpha=-u \alpha^{*}$ or $\pi+u \alpha^{*}$, and $\lambda=0$, with $\alpha^{*}=\alpha^{*}(\ell)=\arcsin \ell$.

Proof. A suitable Hamiltonian is $-\lambda(\ell u+\sin \alpha)$.

The subsystem in $\left(\theta, p_{\theta}\right)$ is also Hamiltonian on $T^{*} S^{1}$ for $\cos (\theta-\varphi)+p_{\theta} u$ but, by analogy with the analysis in the case without trailer, we prefer to integrate as follows:

Lemma 13. Along bang arcs, $\left(p_{\theta}, \dot{p}_{\theta}\right)$ belongs to the union of the two unit circles

$$
\left(p_{\theta}-c_{ \pm}\right)^{2}+\dot{p}_{\theta}^{2}=1
$$

whose centers abscissa are $c_{ \pm}=u \gamma+p_{\alpha}(1+(u / \ell) \sin \alpha)$, $u= \pm 1$.

Proof. Using $H=0$, one obtains

$$
u p_{\theta}-\gamma-p_{\alpha}(u+(1 / \ell) \sin \alpha)=-\cos (\theta-\varphi),
$$

where $p_{\alpha}(u+(1 / \ell) \sin \alpha)$ is constant along bang arcs (see Lemma 12. Noting $\dot{p}_{\theta}=\sin (\theta-\varphi)$ allows to conclude.

Combining the two previous lemmas and adding one quadrature to retrieve $x$ and $y$, we prove that

Proposition 14. The regular flow is Liouville integrable.

Theorem 15. The minimum time extremal flow of the ZMD system with trailer is Liouville integrable.

Proof. In the case $\left(p_{x}, p_{y}\right) \neq(0,0)$, there are only order one singular arcs by Proposition 7 , and Propositions 10 and 14 imply that both regular (bang) and these singular arcs are integrable. In the case $\left(p_{x}, p_{y}\right)=(0,0)$, there are only bang arcs so integrability also holds (Propositions 14 remains valid).

\section{CONCLUSIONS AND FUTURE WORKS}

In this paper we developed a framework for productivity optimization in the marine navigation for seismic acquisition. The framework relies on optimal control. In this context we showed the reachability of the desired configurations and integrability of the extremal flow. A natural follow-up is to establish the exact structure of the optimal flow in terms of singular and regular arcs. Besides, other models could be investigated as for instance models with more than one element to approximate the shape of the real towed underwater cables.

\section{REFERENCES}

[1] A. A. Agrachev and Y. L. Sachkov. Control theory from the geometric viewpoint, volume 87 of Encyclopaedia of Mathematical Sciences. Springer-Verlag, Berlin, 2004. Control Theory and Optimization, II.

[2] E. Bakolas and P. Tsiotras. Optimal synthesis of the Zermelo-MarkovDubins problem in a constant drift field. J. Optim. Theory Appl., 156(2):469-492, 2013.

[3] J.-D. Boissonnat, A. Cérézo, and J. Leblond. Shortest paths of bounded curvature in the plane. J. Intell. Robot. Syst., 11:5-20, 1994.

[4] B. Bonnard and M. Chyba. Singular trajectories and their role in control theory, volume 40 of Mathématiques \& Applications. SpringerVerlag, Berlin, 2003.

[5] J.-M. Coron. Control and nonlinearity, volume 136 of Mathematical Surveys and Monographs. American Mathematical Society, Providence, RI, 2007.

[6] L. E. Dubins. On curves of minimal length with a constraint on average curvature, and with prescribed initial and terminal positions and tangents. American Journal of Mathematics, 79:497-516, 1957.

[7] Int. Assoc. of Oil \& Gas Producers (OGP) and Int. Assoc. of Geophysical Contractors (IAGC). An overview of marine seismic operations. Report 448, IOGP, Apr. 2011.

[8] F. Jean. The car with $N$ trailers: characterisation of the singular configurations. ESAIM Control Optim. Calc. Var., 1:241-266, 1996.

[9] T. G. McGee and J. K. Hedrick. Optimal path planning with a kinematic airplane model. J. Guid. Control Dyn., 30:629-633, 2007.

[10] R. M. Murray and S. S. Sastry. Nonholonomic motion planning: Steering using sinusoids. IEEE Trans. Automat. Control, 38(5):700713, 1993.

[11] H. J. Sussmann and G. Tang. Shortest paths for the Reeds-Shepp car: a worked out example of the use of geometric techniques in nonlinear optimal control. Technical Report SYCON-91-10, Rutgers University, NJ, USA, Sept. 1991.

[12] L. Techy and C. A. Woolsey. Minimum-time path planning for unmanned aerial vehicles in steady uniform winds. J. Guidance, Control, and Dynamics, 32:1736-1746, 2009.

[13] J.-B. Caillau, S. Maslovskaya and J.-B. Pomet. Minimum time optimal control problem in marine navigation. In preparation. 